\title{
Nephrotic Syndrome Associated with Fibrillary Deposits in the Glomeruli
}

\author{
Wako Yumura, Kosaku NitTa, Shigeru Horita, Hiroyuki Ozu, Kazuho Honda and Hiroshi NiHeI
}

\begin{abstract}
A 48-year-old woman with nephrotic syndrome underwent renal biopsy. Light microscopy showed nodular sclerosis and thickening of the glomerular capillary wall. Immunoglobulins (Ig) G, especially IgG2, IgM, IgA, C3, and C1q were detected along the glomerular capillary walls by immunofluorescent microscopy. Electron microscopy revealed that fibrillar materials of about 25 $\mathrm{nm}$ were accumulated in the subepithelial area of the glomerular basement membrane. These materials were negative for Congo-red staining. Neither cryoglobulinemia nor paraproteinemia including light chains was found. This case was diagnosed as membranous nephropathy by clinical findings and pathological examinations, and seemed to be a case of fibrillary glomerulonephritis. (Internal Medicine 34: 46-50, 1995)
\end{abstract}

Key words: renal biopsy, membranous nephropathy, fibrillary glomerulonephritis

\section{Introduction}

Congo red-negative fibrillar deposits in the kidney have previously described $(1,2)$. Using electron microscopy, Alpers et al (1) described fibrillary glomerulonephritis as random fibrillar material in the mesangium and capillary walls that stains Congo-red-negative. Fluorescent microscopy of this fibrillar material shows granular deposits of immunoglobulin and complement in the same distribution. Similarly, Korbet et al (2) reported immunotactoid glomerulopathy with pathologic features identical to those found in fibrillary glomerulonephritis. However, etiology of this glomerulopathy is still unknown.

We present a case of 48-year-old woman with nephrotic syndrome associated with fibrillar deposits in the glomeruli. This case is unique because of positive staining of immunoglobulins (Ig), especially IgG2 and complements in the glomeruli.

\section{Case Report}

A 48-year-old Japanese woman was detected to have microscopic hematuria in April 1988, and had been followed by a physician for 6 months when urinalysis gave positive tests for protein as well as red blood cells. She was referred to the Kidney Center of Tokyo Women's Medical College at the end of 1988. Her urinary protein excretion tended to increase in amount, and consequently pretibial edema was noted in January 1989. The clinical course of this case is summarized in Fig. 1. She was admitted to our hospital because of massive proteinuria and underwent the first renal biopsy. Unfortunately, the size of the specimen was too small to obtain decisive pathological diagnosis, since the specimen contained only one glomerulus. A focal fibrotic change was observed in the interstitium, and the glomerulus manifested peculiar fibrillar deposits in the subepithelial area. Therapy was commenced with $40 \mathrm{mg} /$ day of prednisolone concomitant with an antiplatelet agent. Although the response to prednisolone was not prompt, daily urinary protein excretion decreased gradually to $0.13 \mathrm{~g} /$ day in eight months (Fig. 1). The dose of prednisolone was then tapered according to the levels of urinary protein, to the alternative dose of $10 \mathrm{mg} /$ day. In the spring of 1992, the urinary protein level seemed to be increasing again, and in May 1992 the urinary protein excretion was above $5 \mathrm{~g} / \mathrm{day}$. A high dose of prednisolone $(50 \mathrm{mg} /$ day) was resumed. The response to steroid therapy was not as good as it had been. An obvious reduction in urinary protein excretion was not observed within 3 months, leading to the indication of the second admission. The patient had no history of alcoholism, exposure to chemicals, or drug abuse. There was no family history of renal disease or systemic diseases. Her blood pressure on admission was $102 / 60 \mathrm{mmHg}$. Physical findings disclosed moderate pretibial leg edema but no other abnormalities including the dysplasia of bone and nail were found.

Laboratory findings were as follows: Urinalysis revealed

From the Department of Medicine, Kidney Center, Tokyo Women's Medical College, Tokyo

Received for publication March 30, 1994; Accepted for publication September 21, 1994

Reprint requests should be addressed to Dr. Wako Yumura, the Department of Medicine, Kidney Center, Tokyo Women's Medical College, 8-1 Kawada-cho, Shinjuku-ku, Tokyo 162 
Case T.S. 48 y. o. female

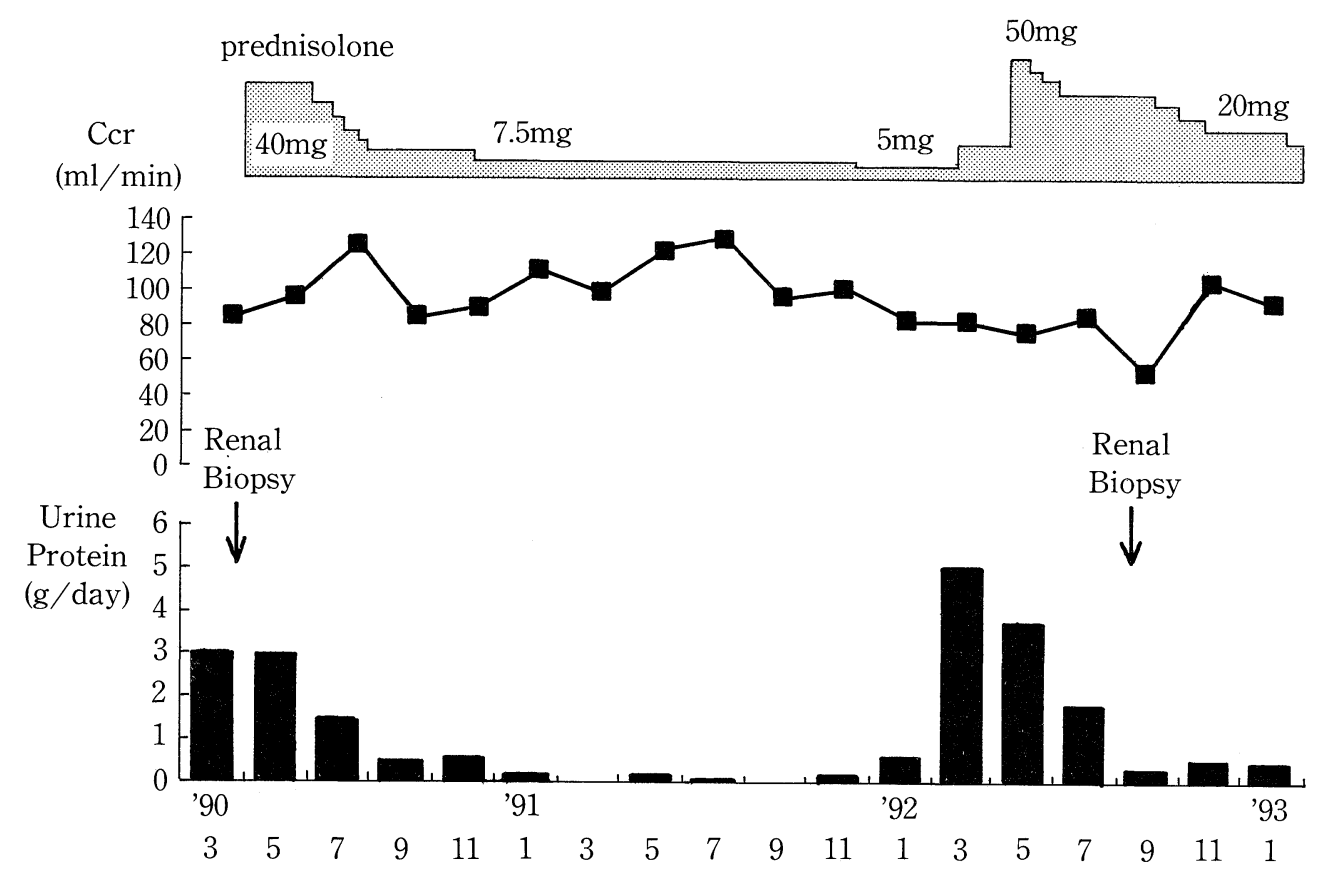

Fig. 1. Clinical course of the patient.

slight proteinuria $(0.43 \mathrm{~g} /$ day $)$ with pathological sediments. Bence Jones protein was not detected in the urine. The hematocrit was $39.3 \%$, hemoglobin $13.0 \mathrm{~g} / \mathrm{dl}$, with normal differentials. Serum total protein was $6.0 \mathrm{~g} / \mathrm{dl}$, albumin $3.8 \mathrm{~g} / \mathrm{dl}$, creatinine $0.9 \mathrm{mg} / \mathrm{dl}$, urea 18.3 and uric acid $4.2 \mathrm{mg} / \mathrm{dl}$. Serum electrolytes were within normal limits. Creatinine clearance $(\mathrm{Ccr})$ was $101.8 \mathrm{ml} / \mathrm{min}$. Blood glucose and Hb-A1 levels were normal. The protein electrophoretic pattern of the serum showed no monoclonal peak. There was no abnormality of the concentrations of serum IgG, IgM, or IgA. Moreover, C3, C5 and CH50 were within normal range. Auto-antibodies were not detected. Chest and abdomen X-rays were normal and electrocardiography showed no abnormal findings. Percutaneous renal biopsy was again performed at our hospital in April 1992.

Renal tissue was processed for light (LM), immunofluorescent (IF) and electron (EM) microscopic examination. The specimens for LM contained 15 glomeruli. Two glomeruli showed global obsolescence. In other glomeruli, nodular lesion (arrow) by Periodic acid-Schiff staining (Fig. 2A), and thickening of the glomerular capillary walls and spike formation (arrowhead) by Periodic acid-methenaine-silver (PAM) staining (Fig. 2B) were found. In some parts of the capillary walls, double contours (arrowhead) were found with PAM staining. Extra-capillary or intra-capillary cell proliferation was not prominent and there was only slight infiltration of inflammatory cells. The renal tubules were focally atrophic. The tissue was negative under polarized light microscopy after staining with Congo red.

By EM, fibrillar materials were observed in the subepithelial areas of glomerular basement membranes, which led to the

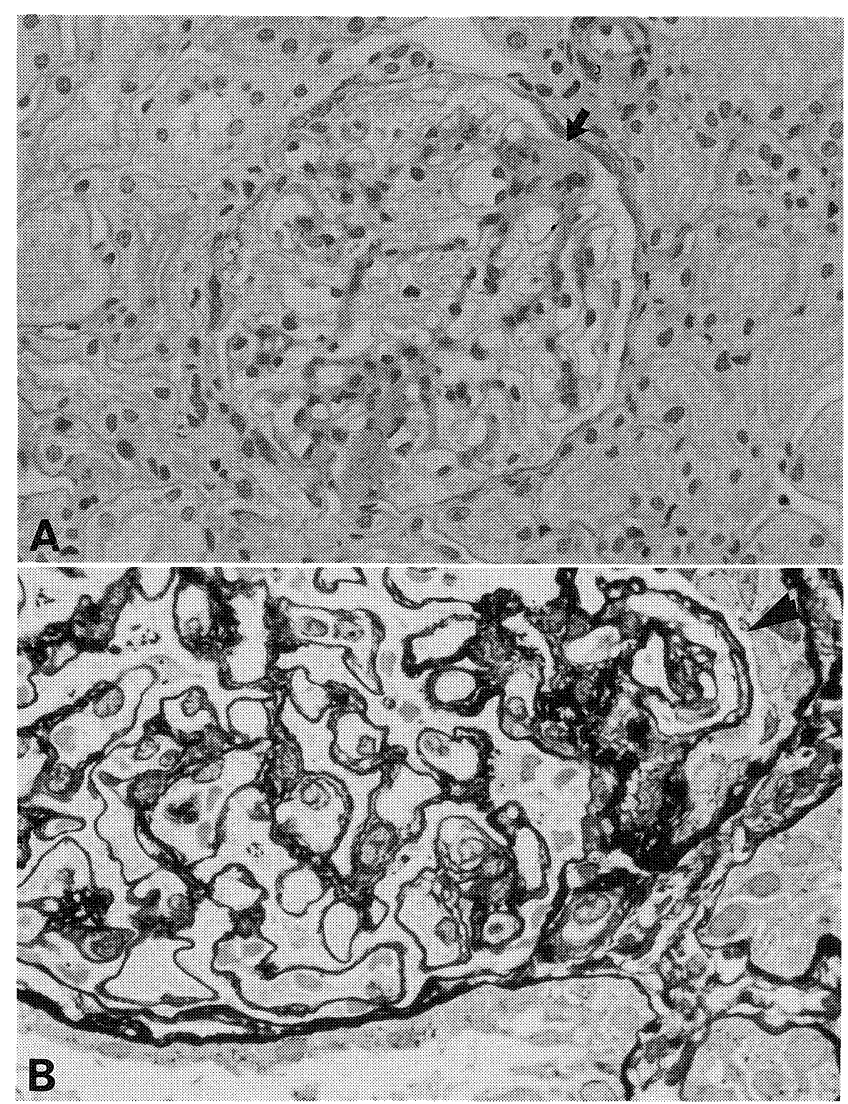

Fig. 2. Light micrographs of the glomerulus showing nodular sclerosis (arrow) with mesangial expansion (A) (PAS stain, $\times 200$ ) and (B) thickening of glomerular capillary walls and double contour (arrowhead) (PAM stain, $\times 200$ ). 


\section{YuMURA et al}

irregular expansion of subepithelial space (Fig. 3A) and mesangial matrix. There were also electron dense deposits in the glomerular basement membrane. On high power field, the fibrillar materials were irregularly curved and about $25 \mathrm{~nm}$ (Fig.
3B).

IF revealed positive IgG (Fig. 4A) and $\mathrm{C} 3$ staining in a partially linear pattern along glomerular capillary walls. As a subtype of $\operatorname{IgG}, \operatorname{IgG} 2$ was strongly positive in the same area of
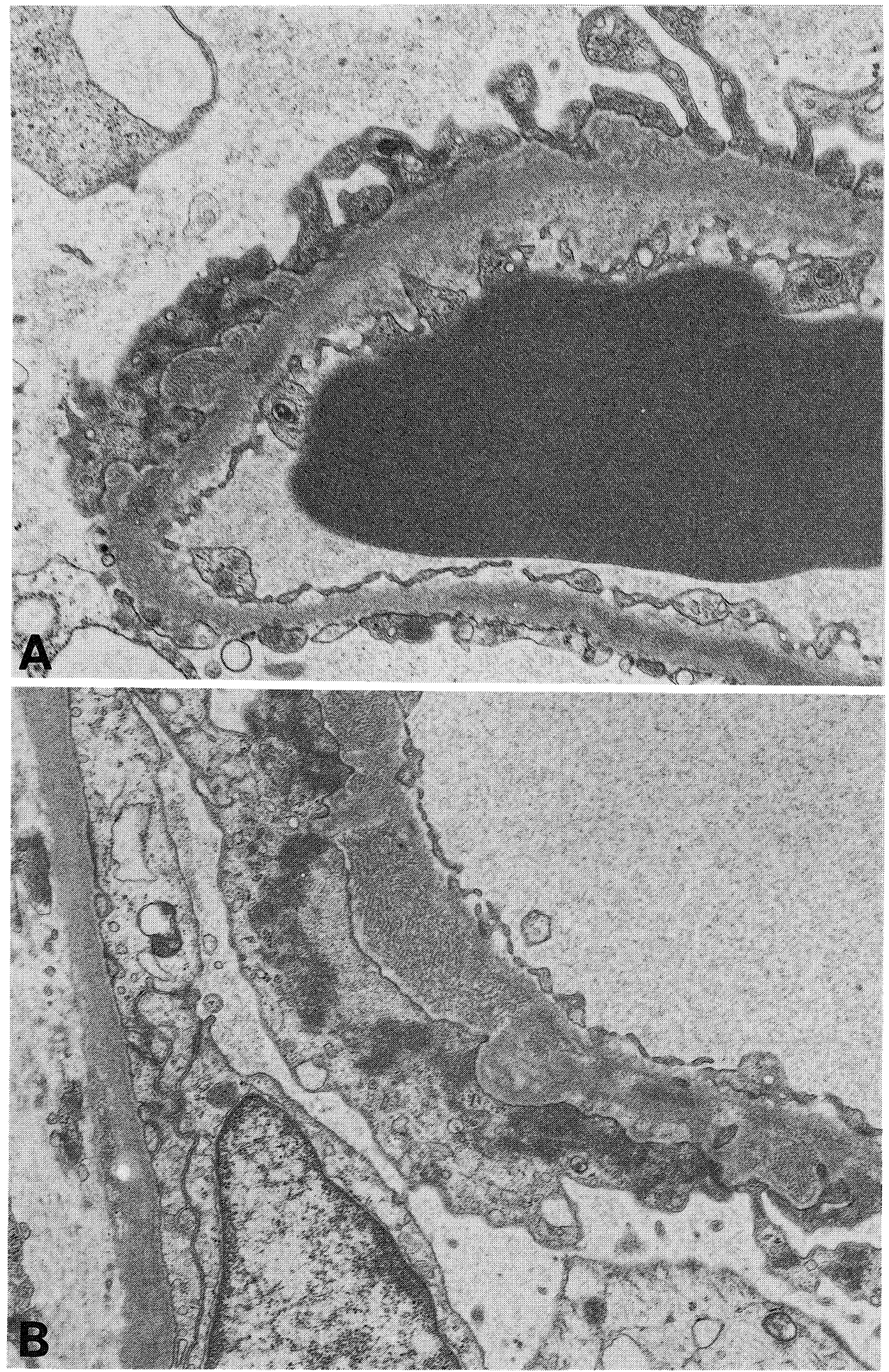

Fig. 3. (A) Electron microscopy shows diffuse deposition of fibrillar material along the subepithelial area of the glomerular basement membranes $(\times 2,500)$. (B) Higher magnification shows distribution and size of fibrils in the subepithelial area $(\times 4,500)$. 


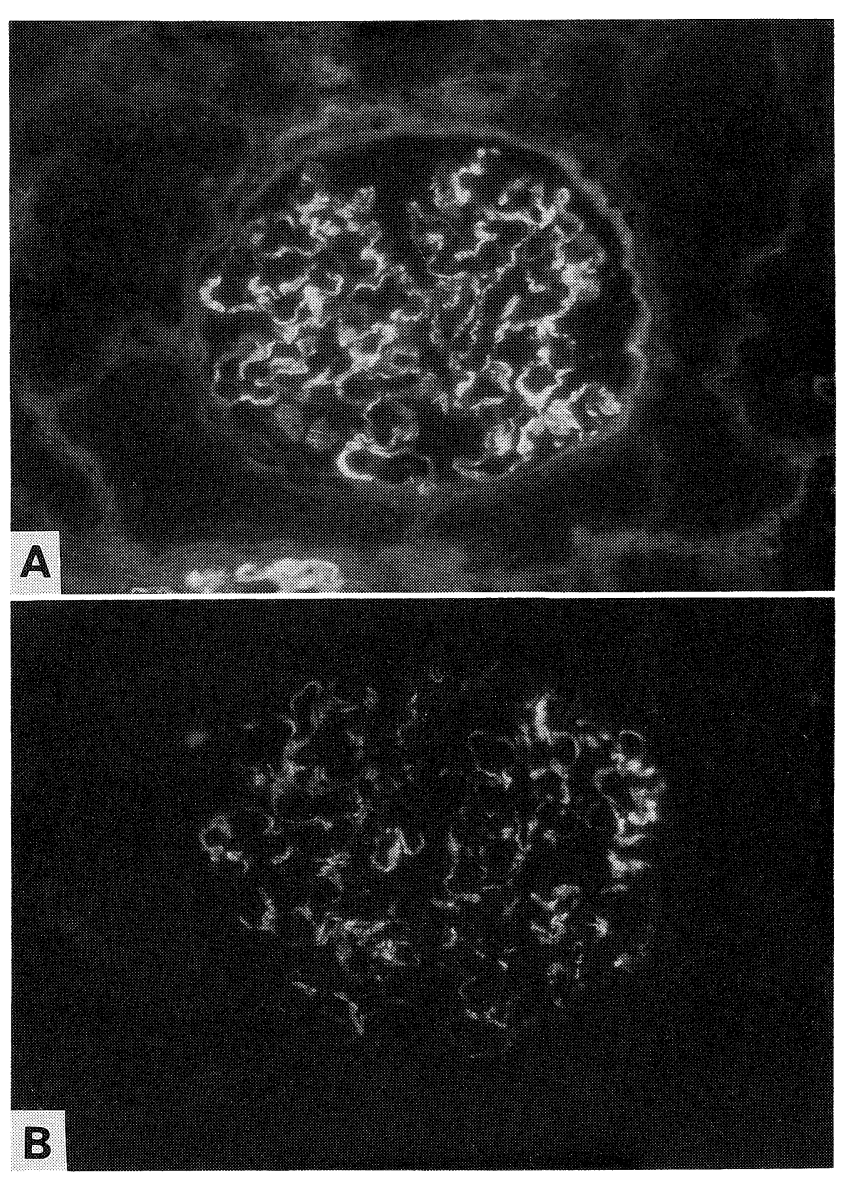

Fig. 4. Immunofluorescence findings show prominent staining of glomerular capillary walls for IgG $(A, \times 200)$, especially IgG2 $(B, \times 200)$ as a subtype.

IgG staining (Fig. 4B), but other subtypes of IgG, IgM and IgA were not detected. In order to clarify the nature of the fibrillar materials, monoclonal antibodies against type I and type III collagen were used. However, the fibrillar regions were negative for these stainings.

At present, the nephrotic state of this patient has not progressed. Urinary protein excretion (24-hour) is less than $0.5 \mathrm{~g} /$ day and serum total protein is $6.0 \mathrm{~g} / \mathrm{dl}$ and albumin $3.8 \mathrm{~g} / \mathrm{dl}$. The renal function is slightly improved at present compared with that of her first admission to our hospital. Blood pressure is 120 / $70 \mathrm{mmHg}$ without antihypertensive agents. Treatment with prednisolone ( $35 \mathrm{mg} /$ day) and dipyridamole $(200 \mathrm{mg} / \mathrm{day})$ was effective for improving the proteinuria. The whole clinical course is shown in Fig. 1.

\section{Discussion}

In the present case, nephrotic syndrome was caused by membranous nephropathy based on the clinicopathological findings. Treatment by the prednisolone was effective for decreasing urinary protein excretion. The second renal biopsy findings revealed a focal segmental sclerosis, thickening of glomerular capillary walls, and partial granular deposition of IgG, IgM, IgA and complements. However, cell proliferation was not prominent in either the intracapillary or extracapillary sites. The most interesting and striking alteration was a deposition of fibrillar materials in the subepithelial area of the glomerular basement membrane. Amyloid fibrils were not present because of the absence of reaction with Congo red, antilight chain (kappa or lamda chain) (3). Collagen fibers were not identical to the fibrillar materials because immunohistochemical staining using monoclonal antibodies against type I and type III collagen was negative. The size of the fibrillar material was about $25 \mathrm{~nm}$ by EM. From these data, the accumulation of fibrillar materials was considered to be of a case of fibrillary glomerulonephritis (1) which is different from amyloidotic nephropathy (4), light chain diseases (5) and collagenofibrotic glomerulonephropathy (6).

Although the presence of extracellular fibrillar material has been observed in a wide variety of glomerulonephritis cases, most often this material has represented collagen, fibrin, or normally occurring components of glomerular basement membrane or mesangial matrix (7-9). The size and random arrangement of the fibrillar material seen in the patients with several types of glomerulonephritis distinguishes it from these other components. The differential diagnosis of lesions in fibrillar glomerulonephritis composed of randomly arranged, non-tubular, large fibrillar structures is restricted to amyloid and amyloid-like lesions that are most often associated with dysproteinemias or cryogloburinemia $(9,10)$.

Both microtubules and microfibrils were suggestive of immunoreactants coming from the blood and contributing to make up preformed or mounted in situ immune complexes. They share in the glomeruli the same seats with granular deposits shown by immunofluorescence and, at least for microfibrils, are combined with granular electron-dense material usually considered the ultrastructural feature of immune complexes (11). Indeed, granular deposits of Ig and complement fractions are detected in all cases of fibrillar glomerulonephritis by immunofluorescence studies (9-11). In the present case, the site of deposition of Ig was identical to that of fibrillar materials. Therefore, fibrillar materials are thought to be associated with Ig. Furthermore, a recent ultrastructural study has shown co-localization of amyloid-P component with polyclonal IgG and $\mathrm{C} 3$ in the fibrillary deposits, suggesting that these fibrils can bind the amyloid-P component (12). These findings suggest that these deposits are not typically monoclonal proteins, but may be contain, or be derived from, IgG molecules $(12,13)$.

Glomerulopathy characterized by deposits of fibrillar material was first reported only 16 years ago (14). This was first thought to be a rare disease. However, as recognition of these lesions has increased, over 100 cases have been reported. Of note, several of these series combined cases with fibrillary deposits, and a wide range of age at presentation and variable incidences of hematuria and proteinuria are evident. End-stage renal disease developed in $42 \%$ of the patients with follow-up information (15). 


\section{YUMURA et al}

Nephrotic syndrome due to membranous nephropathy in the present case was responsive to prednisolone therapy. Moreover, renal function was not deteriorated for 3 years from the onset of nephrotic syndrome. According to the data of Alpers et al (1), renal failure occurs within 1 or 2 years of diagnosis, a rather short period which made the favorable prognosis in the case reported by Mazzucco et al (11) with shorter follow-up reliable. On the other hand, the better prognosis of their case does not seem to be due to the therapy, since there are not significant differences among treated and untreated cases. Considering data of the present case and others, fibrillary glomerulonephritis might be a heterogenous disorder.

As discussed by Korbet et al $(2,16)$, it is likely that intrinsic characteristics of the immunoreactant or some environmental conditions can lead to this organization of deposits which could well represent only a peculiar morphologic marker. Nevertheless, their biological and clinical significance is at present uncertain. At present, further investigations and a greater number of cases are necessary in spite of its status of a "new entity" as described by Alpers et al (1).

\section{References}

1) Alpers CE, Rennke H, Hopper J Jr, Biava CG. Fibrillary glomerulonephritis: An entity with unusual immunofluorescence features. Kidney Int 31: 781, 1987.

2) Korbet SM, Schwartz MM, Rosenberg BF, Sibley RK, Lewis EJ. Immunotactoid glomerulopathy. Medicine 64: 228, 1985.

3) Glenner GG. The bases of the staining of amyloid fibers: Their physiochemical nature and the mechanism of their dye-substrate interac- tion. Prog Histochem Cytochem 13(3): 1, 1981.

4) Kyle RA, Greip PR. Amyloidosis (AL): clinical and laboratory features in 229 cases. Mayo Clin Proc 58: 665, 1983.

5) Alpers CE, Hopper J, Biava CG. Light-chain glomerulopathy with amyloid-like deposits. Hum Pathol 15: 444, 1984

6) Arakawa M, Yamanaka N. Collagenofibrotic glomerulonephropathy. In: Collagenofibrotic Glomerulonephropathy, Arakawa M, Yamanaka N, Eds. Nishimura Co., Ltd., Niigata, 1991, p.3.

7) Hsu HC, Churg J. Glomerular microfibrils in renal disease: A comparative electron microscopic study. Kidney Int 16: 497, 1979.

8) Yoshikawa N, Cameron AH, White RHR, Standring DM. Microfibrils in glomerulopathies of children: An ultrastructural study. J Pathol 136: 123, 1982.

9) Duffy JL, Churg J. Extracellular fibrils in the renal glomerulus. Ultrastruct Pathol 6: 3, 1984.

10) Iskandar SS, Falk RJ, Jennette JC. Clinical and pathologic features of fibrillary glomerulonephritis. Kidney Int 42: 1401, 1992.

11) Mazzucco G, Casanova S, Donini U, Rollino C, Zucchelli P, Piccoli G, Monga G. Glomerulonephritis with organized deposits: A new clinicopathological entity? Light-, electron-microscopic and immunofluorescence study of 12 cases. Am J Nephrol 10: 21, 1990.

12) Yang GCH, Nieto R, Stachura I, Gallo GR. Ultrastructural immunohistochemical localization of polyclonal $\mathrm{IgG}, \mathrm{C} 3$, and amyloid$\mathrm{P}$ component on the Congo red-negative amyloid-like fibrils of fibrillar glomerulonephritis. Am J Pathol 141: 409, 1992.

13) Casanova S, Donini U, Zucchelli P, Mazzucco G, Monga G, Linke RP. Immunohistochemical distribution between amyloidosis and fibrillary glomerulonephritis. Am J Clin Pathol 97: 787, 1992.

14) Rosenmann E, Eliakim M. Nephrotic syndrome associated with amyloidlike glomerular deposits. Nephron 18: 301, 1977.

15) Fogo A, Qureshi N, Horn RG. Morphologic and clinical features of fibrillary glomerulonephritis versus immunotactoid glomerulopathy. Am J Kidney Dis 22: 367, 1993.

16) Korbet SM, Schwartz MM, Lewis EJ. Immunotactoid glomerulopathy. Am J Kidney Dis 17: 247, 1991. 